\title{
Electroencephalographic Rhythmic Delta Activities in Three Cases
}

\author{
(1) Dilara MERMi DiBEK, (1) İbrahim ÖZTURA, () Barış BAKLAN
}

Department of Neurology, Clinical Neurophysiology, Dokuz Eylül University Faculty of Medicine, İzmir, Turkey

\begin{abstract}
Intermittent rhythmic delta activities are electroencephalographic discharges with uniform morphology, at a frequency of $4 \mathrm{~Hz}$ or less, containing at least six cycles, lost by eye opening and stimulation. These are called "plus" if concomitant theta or higher frequency rapid activity or spike, sharp wave activity is present. This group has a stronger relationship with epileptiform abnormality. We aimed to discuss the clinical presentations, and nomenclature of intermittent rhythmic delta activities with three case reports.
\end{abstract}

Keywords: Delta; intermittent; plus; rhythmic; spike.

Cite this article as: Mermi Dibek D, Öztura I, Baklan B. Electroencephalographic Rhythmic Delta Activities in Three Cases. Epilepsi 2021;27:195-200.

\section{Giriş}

Elektroensefalografi (EEG) incelemesinde $4 \mathrm{~Hz}$ veya daha düşük frekanslı, uniform morfolojide, en az altı siklus içeren ( $1 \mathrm{~Hz}$ ise 6 saniye, $3 \mathrm{~Hz}$ ise 2 saniye süreyle) deşarjlar intermittent ritmik delta aktiviteleri (IRDA) olarak adlandırılırlar. [1] Tanımlama ilk defa Cobb ${ }^{[2]}$ tarafından 1945 yılında yapılmıştır. Genellikle bilateral olmak üzere asimetrik veya unilateral kayıtlanabilirler. Yerleşim alanlarına göre alt gruplara ayrılırlar. Ayrıca eşlik eden teta veya daha yüksek frekansta hızlı aktivite veya diken, keskin dalga aktivitesi varlığında "IRDA artı" grubu olarak adlandırılmakta ve bu grubun önemi epileptiform anormallikle ilişkisinin daha yüksek oranda olmasıdır. ${ }^{[1]}$

Kliniğimizde takip edilen üç olgu ile elektrofizyolojik olarak IRDA artı kayıtlanan hastaları klinikleri ile birlikte tartışmayı hedeflemekteyiz.

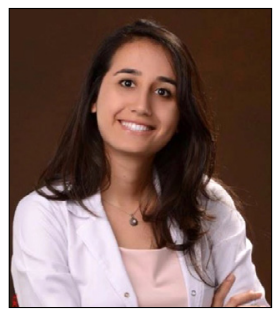

Corresponding author Dilara MERMi DiBEK, M.D.

e-mail dilara_mermi@hotmail.com

Received 29.12.2019

Accepted 28.12.2020

Online date 02.08 .2021

Content of this journal is licensed under a Creative Commons Attribution-NonCommercial 4.0 International License.

Dilara MERMI DIBEK, M.D.

\section{Olgu Sunumu}

Olgu 1- Yirmi beş yaşında, sağ el dominans, ortaokul mezunu, bekar kadın hasta; ataklar halinde gelen baş dönme ve düşme hissi ile kliniğimize başvuruyor. Hastanın şikayetleri yaklaşık 3 yıl önce başlamış, başvurduğu merkezde epizodik seyir nedeniyle antiepileptik tedavi başlanmış. Hastaya başlanan levetirasetam ve zonisamid tedavisine yanıt alınmaması üzerine lakozamid tedavisine geçilerek, hasta kliniğimize lakozomid 100 mg/gün tedavisi kullanmakta iken başvurdu. Özgeçmişinde romatoid artrit ve göz tutulumu (üveit) olan hastanın kortikosteroit kullanımı mevcut iken baş dönmesi ve düşme atakları nedeniyle sistemik kortikosteroit tedavisi kesilmiş ve şikayetlerinde belirgin azalma saptanmış. Ek olarak anksiyete bozukluğu nedeniyle duloksetin $30 \mathrm{mg} /$ gün kullanımı olan hastanın febril konvüziyon öyküsü, kafa travma öyküsü yoktu. Ailede epilepsi veya genetik hastalık öyküsü yoktu. Fizik muayenede cushingoid görünümü olan hastada aydede yüz ve flushing mevcut; sağ göz rekürren üveit atakları nedeniyle \%20 görüyor; sol göz görmesi tam; diğer nörolojik muayenesi normaldi. Hastanın kranial manyetik rezonans görüntülemesi normal olup EEG incelemesinde oksipital ve parietal alanlarda daha çok olmak üzere frontal ve temporal alanlarda ve jeneralize olarak kayıtlanan, 1-9 saniye süreli, $2.5-4 \mathrm{~Hz}$ frekansında intermittent ritmik delta aktiviteleri izlendi. Bu aktivitelere tekli, ikili veya üçlü diken aktivitesinin eşlik ettiği dikkati çekti (Şekil 1).

Hasta; epizodik, kısa süreli (saniyeler) otonomik nitelikteki şikayetlerinin lakozamid dozu arttırıldığında azaldığını ve kortikosteroitin sistemik kullanımında şikayetlerinde artış olduğunu belirtmekteydi. Nöropsikolojik test bataryasında, 


\section{Üç Olgu Sunumu ile Ritmik Delta Aktiviteleri}

$\ddot{0} z$

Elektroensefalografi incelemesinde $4 \mathrm{~Hz}$ veya daha düşük frekanslı, uniform morfolojide, en az altı siklus içeren deşarjlar intermittent ritmik delta aktiviteleri (IRDA) olarak adlandırılırlar. Gözaçma ve stimulus ile kaybolurlar. Eşlik eden teta veya daha yüksek frekansta hızlı aktivite varlığında veya diken, keskin dalga aktivitesi eşlik ettiğinde "IRDA artı" grubu olarak adlandırılmakta ve bu grubun önemi epileptiform anormallikle ilişkisinin daha yüksek oranda olmasıdır. Üç olgu sunumu ile intermitent ritmik delta aktivitelerinin isimlendirilmesini, etiyolojisini ve morfolojilerini tartışmayı amaçladık.

Anahtar sözcükler: Artı; delta; diken; intermittent; ritmik.

kısa süreli sözel bellek testinde kritere (15 kelime) ulaşmış olmasına rağmen kısa süreli sözel öğrenme puanı yaş ve eğitim normlarının altında, dikkate sekonder bellek bozukluğu tespit edilmişti.

Hastanın tekrarlayan EEG izlemlerinde benzer nitelikte IRDA artı aktivitelerinin yanı sıra izole diken yavaş dalga aktivitesi saptandı (Şekil 1).
Saptanan IRDA deşarjları içerisinde TIRDA ve sol posterior temporal alanda izole diken yavaş dalga aktivitesi saptanması, lakozomid tedavisinin tedricen arttırılmasıyla hastanın epizodik ataklarının oldukça azalması nedeniyle olası temporal otonomik bir nöbet olabileceği düşünüldü; tedaviye eklenen $50 \mathrm{mg} / \mathrm{gün}$ lamotrijin tedavisi ile hastanın son 3 aydır atakları olmadı.

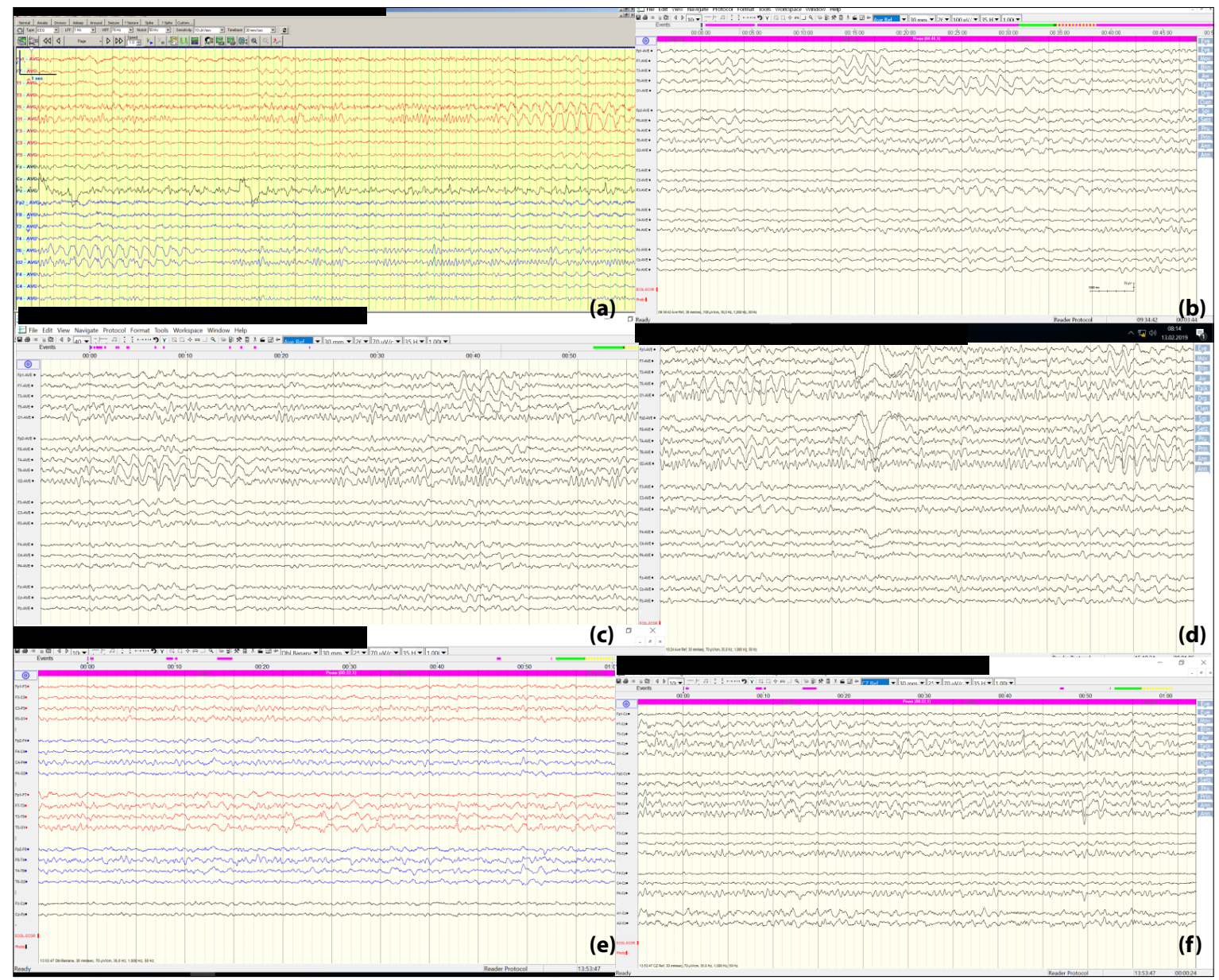

Şekil 1. (a) Bilateral bağımsızT-O RDA; (b) SolTIRDA, SolTPO RDA; (c) Sağ TIRDA+Diken; (d) Bilateral bağımsızT-O RDA+Diken; (e) Sol posterior temporal izole diken yavaş dalga aktivitesi,bipolar longitudinal montaj; (f): Sol posterior temporal izole diken yavaş dalga aktivitesi,monopolar montaj. 
Olgu 2- Yirmi dokuz yaşında, sağ el dominans, kadın hasta, üniversite mezunu. Çocukluk çağında başlayan ataklarının öncesinde sağ kolda uyuşma hissettiğini ardından sağ kolda kasılmayı takiben bilinç kaybı ile iki taraflı kasılma oluyormuş. Hastanın hipoksik doğum öyküsü mevcut, febril konvülziyon öyküsü yok; mental ve motor gelişimi, sistemik ve nörolojik muayenesi normal, ailede epilepsi öyküsü yoktu. Haftada iki veya üç defa atakların devam etmesi nedeniyle başvurduğu merkezde EEG incelemesinde hiperventilasyon sırasında $3 \mathrm{~Hz}$ frekansında diken yavaş dalga aktivitesi olarak değerlendirilen aktivite ve kranial manyetik rezonans görüntüleme normal olması ile valproik asit tedavisi başlanmış ancak sağ kolda uyuşma ve ardından kasılmaları haftalık olmaya devam etmiş. Hastanın nöbetlerinin devam etmesi üzerine merkezimize başvurusunda, nöbet semiyolojisi anamneze göre fokal başlangıçlı olup dış merkezdeki primer jeneralize elektroensefalografik bulgu uyumsuzluğu nedeniyle nörogörüntüleme ve EEG tekrarlandığında, normal olarak saptandı. Reprodüktif çağda kadın olması nedeniyle tedavi levetirastam geçilerek tolere edebildiği 2500 mg/gün dozuna rağmen klinik yanıtsızlık ile lamotrijin tedaviye eklendi, nöbet sıklığında azalma olmaması üzerine hasta bir gecelik video EEG incelemesine alındı. Çekim süresince nöbeti olmayan hastanın, göz kapalı veya erken uyku döneminde senkron veya asenkron olarak 2 saniyeden kısa sürelerle, $3 \mathrm{~Hz}$ frekansında, oksipital ritmik delta aktivitesi kayıtlandı, bu aktiviteye tekli diken aktivitesinin eşlik ettiği saptandı (Şekil 2).
Hastanın nöbet süresinin uzun olması, iki taraflı kasılma olarak tanımladığı dönemlerin uzun süreli ve bir kısmında farkındalığının etkilenmemesi, düşmeleri sırasında korunaklı olarak düşmeleri epileptik olmayan ataklar kuşkusu uyandırması nedeniyle hastada nöbetinin görülmesi amacıyla uzun süreli video EEG incelemeye alınması planlansa da hastanın istememesi ile çekim gerçekleştirilememiştir.

Hastanın anamnezi ile epileptik-nonepileptik nöbet ayrımı yapılamamış, ev video kaydı elde edilememiş, elektroensefalografi incelemesinde kayıtlanan OIRDA ve OIRDA + diken aktiviteleri nöbet sınıflaması için yeterli olmamıştır. Hastanın ataklarının uzun video EEG veya ev video kaydı ile nöbetlerinin görülmesi ile ayırt edilmesi gerekmektedir.

Olgu 3- Yirmi sekiz yaşında, sağ el dominans, erkek hasta, 4 yaşında kafa travması sonrası meydana gelen nöbetleri özellikle gün ışığında artmaktaymış. Hastanın nöbetleri özensiz boş bakmalarının olduğu ve eşlik eden oroalimenter otomatizmalarla birlikte bilateral tonik klonik nöbetlere evriliyormuş. Hastanın doktor başvurusunda başlanan antiepileptik tedavi ile fokal başlangıçlı bilateral tonik klonik nöbetlerinin sonlandığı ancak yılda 1-2 defa farkındalığının kaybolduğu, boş bakma ve oroalimenter otomatizmalarının eşlik ettiği nöbetleri karbamazepin $1000 \mathrm{mg} / \mathrm{gün}$, topiramat $400 \mathrm{mg} / \mathrm{gün}$, levetirasetam $500 \mathrm{mg} /$ gün tedavisine rağmen devam etmekteymiş. Lise mezunu ve çalışmayan

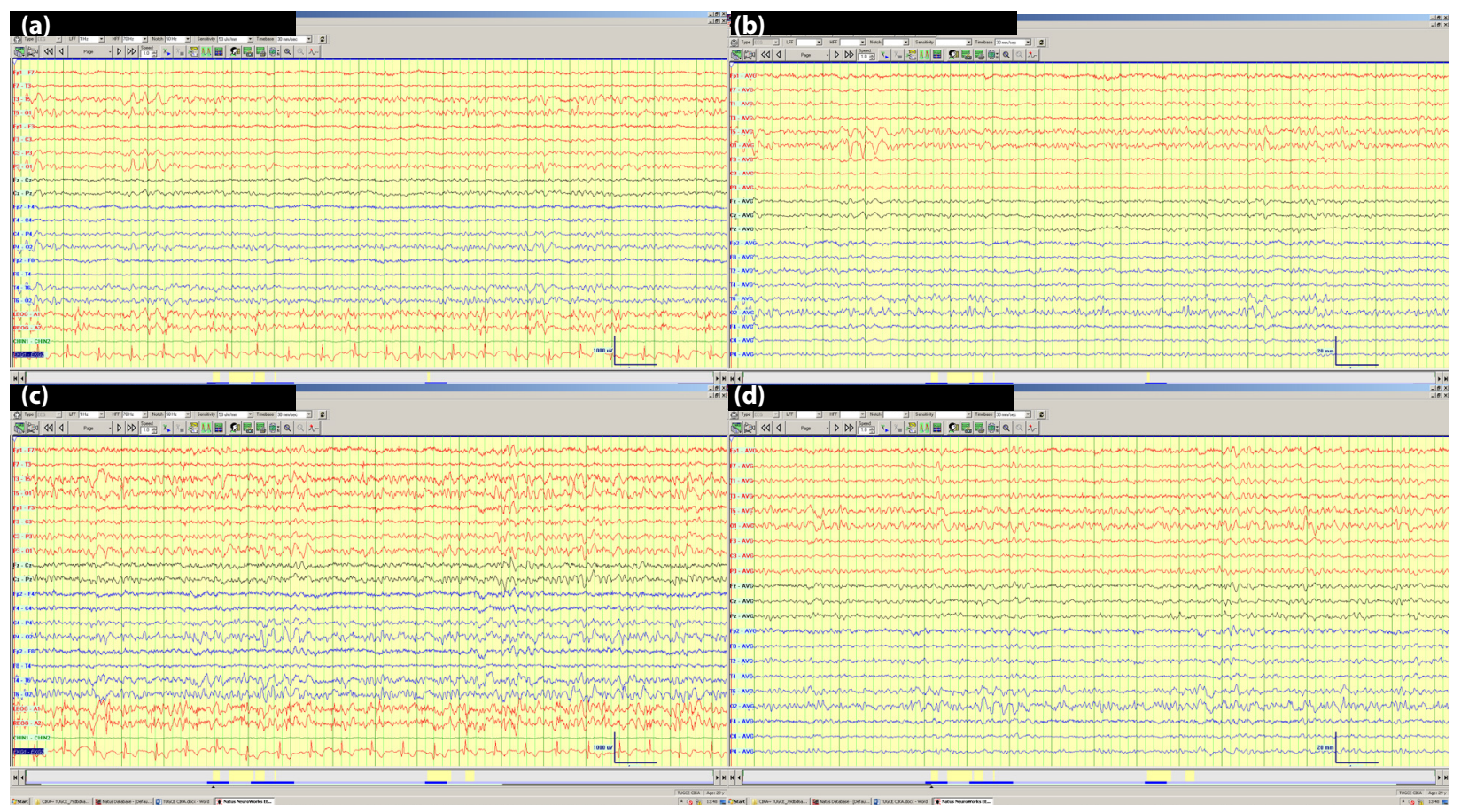

Şekil 2. (a) Sol OIRDA+diken, bipolar longitudinal montaj; (b) Sol OIRDA+diken, monopolar montaj; (c) Bilateral OIRDA+diken bipolar longitudinal montaj; (d) Bilateral OIRDA+diken, monopolar montaj. 


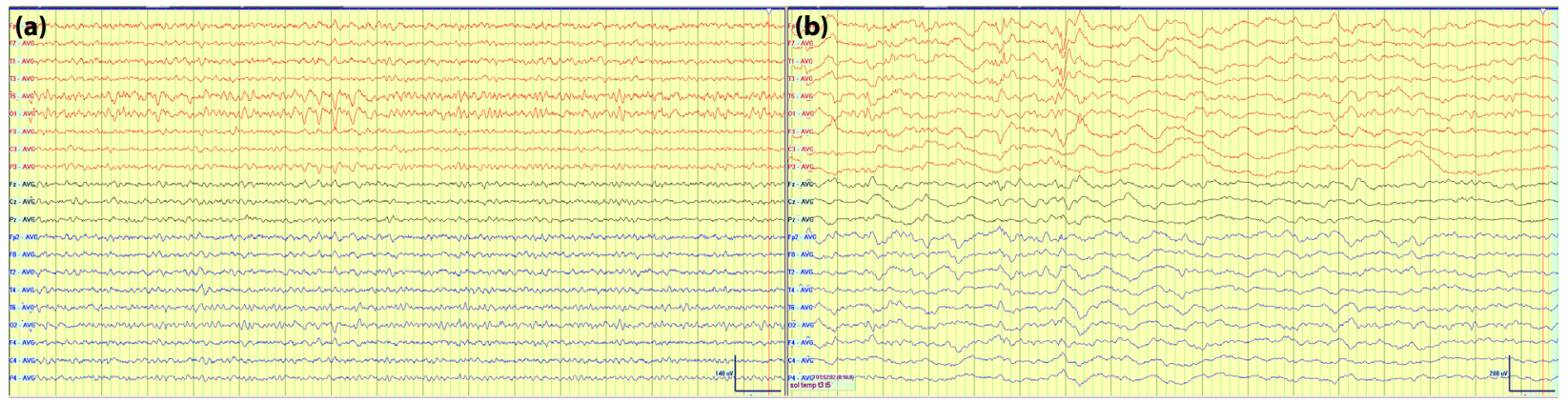

Şekil 3. (a) Sol OIRDA+diken, (b) NREM uyku evresinde sol anterior temporal alanda kayıtalann diken yavaş dalga aktivitesi.

hastanın özgeçmişinde doğum komplikasyon öyküsü ve febril konvülziyon öyküsü yoktu. Anne ve baba arasında kan bağı bulunmayan hastanın büyük ağabeyinde nokturnal başlangıcı bilinmeyen tonik klonik nöbetlerle seyirli EEG incelemesinde temporal interiktal odak ile uyumlu epilepsi öyküsü mevcuttu. Sistemik ve nörolojik muayenesi normal olan hasta antiepileptik tedaviye dirençli fokal nöbet olması üzerine gerçekleştirilen video EEG incelemesinde uyku ile artan sol anterior temporal (F7-T1) irritatif odak tespit edildi. Ek olarak göz kapalılığı süresinde bilateral senkron veya asenkron diken aktivitesinin eşlik ettiği oksipital ritmik delta aktiviteleri saptandı (Şekil 3).

Nöbet semiyolojisi ve elektroensefalografi bulgusu ile temporal lop epilepsi grubunda sınıflandırılan hastanın EEG kaydında gözlenen interiktal sol anterior temporal fokal diken yavaş dalga aktivitesi yanında saptanan OIRDA+diken aktivitesi nadir de olsa erişkin yaşta ve fokal bir epilepside saptanabileceğine uygun bir örnektir.

\section{Tartışma}

Intermittent ritmik delta aktiviteleri (IRDA) $1945^{\prime}$ te Cobb ${ }^{[2]}$ tarafından tanımlanmıştır. Lokalizasyon alanına göre frontal, temporal, occipital, parietal ve jeneralize olarak altgruplara ayrılmaktadır. Nöbet ile ilişkileri ve eşlik ettikleri nöbet özellikleri ile birbirlerinden farklılık göstermektedirler.

FIRDA olarak 1959 'da VanderDrift ve Magnus ${ }^{[3]}$ tarafından tanımlanan frontal ve frontopolar alanlarda lokalize 1.5-4 $\mathrm{Hz}$ frekansında intermittent ritmik delta aktiviteleri, sıklıkla erişkin yaş grubunda görülür. Orta hat veya derin serebral lezyonlarda, arka fossa ve üçüncü ventrikül yapısal lezyonlarında, subkortikal lezyonlar, serebral ödem, üremi veya karaciğer yetmezliği kaynaklı metabolik ensefalopatilerde, baziller arter migrenlerinde, kortikobazal dejenerasyon ve progresif supranuklear palside, Creutzfeld-Jacob hastalığı erken döneminde, Lewy cisimcikli demans, salmonella ensefalopatisi olgularında bildirilmiş, yapısal lezyonlar ve ensefalopatilerde bağımsız olarak ilişkili bulunmuştur. ${ }^{[4-17]}$ Nor- mal bireylerde de hiperventilasyon sırasında hipokapninin tetiklemesi sonucu saptanabilmektedir. ${ }^{[18]}$ FIRDA'ların epileptiform aktivite ile ilişkisi hastaların \%2'sinin altında saptansa da genellikle epileptik bir patern olarak düşünülmemektedir. Literatürde unilateral kayıtlandıklarında lezyon ipsilateral alanda olmayabileceği bilinse de son bildiriler unilateral olduklarında lezyonla ipsilateral olabileceği ancak bilateral FIRDA varlığının yapısal lezyondan kaynaklandığını ekarte etmeyeceği belirtilmektedir. ${ }^{[19]}$

Reiher ${ }^{[20]}$ tarafından tanımlanan, temporal bölgede kayıtlanan intermittent ritmik delta aktivitesi ise TIRDA olarak adlandırılır. Testere dişi veya sinüzoidal morfolojide, ritmik, intermittent, 1-4 Hz frekansında, 50-100 $\mu \mathrm{V}$ amplitüdde anterior temporal montajlarda daha belirgin olarak kayıtlanır. [20] TIRDA, temporal diken veya keskin yavaş dalga aktiviteleri gibi interiktal temporal epilepsi ile yakından ilişkilidir; OIRDA ve FIRDA'nın aksine kayıtlandığı montajın ipsilateral patolojisine işaret etmektedir. ${ }^{[20]}$ Otoimmün hastalığı olan ve immün supresan tedavi almak durumunda olan ilk olgumuzun tanımladığı nonspesifik denebilecek bulgularının kısa süreli ve otonomik nitelikte olması, verilen antiepileptik tedaviye yanıt alması, saptanan intermittent ritmik delta aktivitesinin içerisinde temporal epilepsi ile ilişkisi bildirilen TIRDA deşarjlarının olması hastanın temporal epilepsi olasılığını kuvvetlendirmiş, izlemde hastanın bulgularının antiepileptik tedaviye yanıt alınması ve inteiktal temporal epileptiform aktivite varlığı da tanıyı desteklemektedir.

Oksipital yerleşimli OIRDA olarak adlandırılan intermittent ritmik delta aktivitesi absans epilepsili hastalarda Cobb tarafından tanımlanmıştı..$^{[1]}$ OIRDA hemen tamamen çocuk çağda görülür ve primer jeneralize epilepsilerin interiktal epileptiform paterni olarak değerlendirilir. ${ }^{[21,22]}$ Göz açma ve uykunun derin evrelerinde yok olurken hiperventilasyon ve uyanıklıktan uykuya geçişte ortaya çıkabilir. ${ }^{[21]}$ Bildirdiğimiz üçüncü olgu ise bu bilgilerin aksine yirmi sekiz yaşında erişkin çağda bir birey ve nöbet semiyolojisi, interiktal elektrofizyolojik bulguları, kullanılan anti-epileptik tedavi yanıtlılığı 
ile temporal epilepsi olup "OIRDA + diken" aktivitesi net bir şekilde tespit edilmiş; bu durum fokal epilepsilerde ve erişkin çağda da OIRDA saptanabileceğine uygun bir örnektir. EEG incelemesinde aktiviteleri tanımlamak hastaların tanılarına yön vermek adına oldukça önemlidir; bipolar montajda lokalize rudimenter diken yavaş dalga izlenimi veren deşarjlar da olsa monopolar bağlantıda oksipitalde lokalize olan ve yavaş delta dalgasının ardından diken eklenmesi deneyimsiz gözlerce $3 \mathrm{~Hz}$ diken yavaş denilerek bir hastaya primer jeneralize tanısı verebilir. İkinci olgumuzun tanımladığı fokal başlangıçı nöbet veya epileptik olmayan ataklarına bir de primer jeneralize elektrofizyolojik yorum eklenince hasta çözümsüz bir hal alabilmektedir. Oysa ki bu deşarjlar OIRDA + diken aktiviteleridir.

2013'te Amerikan Klinik Nörofizyoloji Derneği'nin sınıflaması ile intermittent ritmik delta aktivitelerine, teta veya daha yüksek frekanslı hızı ıktivite veya diken aktivitesinin eşlik etmesi " $+S$ (Spike) / F (Fast) " olarak tanımlanmakta ve literatürde IRDA artı grubunun epileptiform anormallik ile ilişkisinin yüksek olduğu ve komadaki hasta grubunda ise kötü prognostik elektrofizyolojik bulgu olduğu belirtilmektedir. ${ }^{[1,23]}$ Bildirdiğimiz olgular ile klinik özellikleri ile IRDA elektrofizyolojk görüntüleri özetlenmiştir.

Informed Consent- Written informed consent was obtained from patients who participated in this study.

Peer-review- Externally peer-reviewed.

Authorship Contributions- Concept: D.M.D., I.Ö., B.B.; Design: D.M.D., I.Ö., B.B.; Supervision: D.M.D., I.Ö., B.B.; Data collection \&/or processing: D.M.D., i.Ö., B.B.; Analysis and/or interpretation: D.M.D., I.Ö., B.B.; Literature search: D.M.D., I.Ö., B.B.; Writing: D.M.D., I.Ö., B.B.; Critical review: D.M.D., I.Ö., B.B.

Conflict of interest- The authors declare that they have no conflict of interest.

Financial Disclosure: The authors declared that this study has received no financial support.

Hasta Onamı- Bu çalışmaya katılan hastalardan yazılı hasta onamı alındı.

Hakem Değerlendirmesi- Dış bağımsız.

Yazarlık Katkıları- Konsept: D.M.D., I.Ö., B.B.; Dizayn: D.M.D., i.Ö., B.B.; Kontrol: D.M.D., I.Ö., B.B.; Veri Toplama veya Iş̧leme: D.M.D., I.Ö., B.B.; Analiz ve Yorumlama: D.M.D., I.Ö., B.B.; Literatür Arama: D.M.D., I.Ö., B.B.; Yazan: D.M.D., I.Ö., B.B.; Kritik Revizyon: D.M.D., I.Ö., B.B.

Çıkar Çatışması- Yoktur.

Finansal Destek- Yazarlar bu çalışma için finansal destek almadıklarını beyan etmişlerdir.

\section{Kaynaklar}

1. Hirsch LJ, LaRoche SM, Gaspard N, Gerard E, Svoronos A, Herman ST, et al. American Clinical Neurophysiology Society's Standardized Critical Care EEG Terminology: 2012 version. J Clin Neurophysiol 2013;30(1):1-27.

2. COBB WA. Rhythmic slow discharges in the electroencephalogram. J Neurol Neurosurg Psychiatry 1945;8(3-4):65-78. [CrossRef]

3. VanderDrift $\mathrm{JH}$, Magnus $\mathrm{O}$. The value of the EEG in the differential diagnosis of cases with cerebral lesions. Electroencephalogr Clin Neurophysiol 1959;11:733-46.

4. Faure J, Droogleever-Fortuyn J, Gastaut H, Larramendi L, Martin $P$, Passouant $P$, et al. Genesis and significance of rhythms recorded at a distance in cases of cerebral tumors. Electroencephalogr Clin Neurophysiol 1951;3(4):429-34. [CrossRef]

5. Daly D, Whelan JL, Bickford RG, Maccarty CS. The electroencephalogram in cases of tumors of the posterior fossa and third ventricle. Electroencephalogr Clin Neurophysiol 1953;5(2):203-16. [CrossRef]

6. Jasper H, Van Buren J. Interrelationship between cortex and subcortical structures: clinical electroencephalographic studies. Electroencephalogr Clin Neurophysiol Suppl 1955;(Suppl 4):168-88.

7. Gastaut JL, Michel B, Hassan SS, Cerda M, Bianchi L, Gastaut H. Electroencephalography in brain edema (127 cases of brain tumor investigated by cranial computerized tomography). Electroencephalogr Clin Neurophysiol 1979;46(3):239-55. [CrossRef]

8. Fariello RG, Orrison W, Blanco G, Reyes PF. Neuroradiological correlates of frontally predominant intermittent rhythmic delta activity (FIRDA). Electroencephalogr Clin Neurophysiol 1982;54(2):194-202. [CrossRef]

9. Pietrini V, Terzano MG, D'Andrea G, Parrino L, Cananzi AR, Ferro-Milone F. Acute confusional migraine: clinical and electroencephalographic aspects. Cephalalgia 1987;7(1):29-37. [CrossRef]

10. Frequin ST, Linssen WH, Pasman JW, Hommes OR, Merx HL. Recurrent prolonged coma due to basilar artery migraine. A case report. Headache 1991;31(2):75-81. [CrossRef]

11. Tashiro K, Ogata K, Goto Y, Taniwaki T, Okayama A, Kira J, et al. EEG findings in early-stage corticobasal degeneration and progressive supranuclear palsy: a retrospective study and literature review. Clin Neurophysiol 2006;117(10):2236-42. [CrossRef]

12. Hansen $H C$, Zschocke $S$, Stürenburg $H J$, Kunze K. Clinical changes and EEG patterns preceding the onset of periodic sharp wave complexes in Creutzfeldt-Jakob disease. Acta Neurol Scand 1998;97(2):99-106. [CrossRef]

13. Wieser HG, Schwarz U, BlättlerT, Bernoulli C, Sitzler M, Stoeck K, et al. Serial EEG findings in sporadic and iatrogenic Creutzfeldt-Jakob disease. Clin Neurophysiol 2004;115(11):2467-78. [CrossRef]

14. Wieser HG, Schindler K, Zumsteg D. EEG in Creutzfeldt-Jakob disease. Clin Neurophysiol 2006;117(5):935-51. [CrossRef]

15. Uysal H, Karademir A, Kilinç M, Ertürk O. Salmonella encephalopathy with seizure and frontal intermittent rhythmic delta activity. Infection 2001;29(2):103-6. [CrossRef] 
16. Calzetti S, Bortone E, Negrotti A, Zinno L, Mancia D. Frontal intermittent rhythmic delta activity (FIRDA) in patients with dementia with Lewy bodies: a diagnostic tool? Neurol Sci 2002;23(Suppl 2):S65-6. [CrossRef]

17. Roks G, Korf ES, van der Flier WM, Scheltens P, Stam CJ. The use of EEG in the diagnosis of dementia with Lewy bodies. J Neurol Neurosurg Psychiatry 2008;79(4):377-80. [CrossRef]

18. Takahashi T. Activation methods: hyperventilation. In: Niedermeyer E, Lopes Da Silva F, editors. Electroencephalography: basic principles, clinical applications, and related fields. Baltimore: Williams \& Wilkins; 1993. p. 241-2.

19. Cerrahoğlu Şirin T, Bekdik Şirinocak P, Arkalı BN, Akıncı T, Yeni SN. Electroencephalographic features associated with intermittent rhythmic delta activity. Neurophysiol Clin 2019;49(3):227-34.
20. Reiher J, Beaudry M, Leduc CP. Temporal intermittent rhythmic delta activity (TIRDA) in the diagnosis of complex partial epilepsy: sensitivity, specificity and predictive value. Can J Neurol Sci 1989;16(4):398-401. [CrossRef]

21. Riviello JJ Jr, Foley CM. The epileptiform significance of intermittent rhythmic delta activity in childhood. J Child Neurol 1992;7(2):156-60. [CrossRef]

22. Guilhoto LM, Manreza ML, Yacubian EM. Occipital intermittent rhythmic delta activity in absence epilepsy. Arq Neuropsiquiatr 2006;64(2A):193-7. [CrossRef]

23. Gungor Tuncer O, Altindag E, Ozel Yildiz S, Nalbantoglu M, Acik ME, Tavukcu Ozkan S, et al. Reevaluation of the Critically III Patients With Nonconvulsive Status Epilepticus by Using Salzburg Consensus Criteria. Clin EEG Neurosci 2018;49(6):425-32. 